\title{
Identification of two signaling submodules within the Crkll/ELMO/Dock180 pathway regulating engulfment of apoptotic cells
}

\author{
A-C Tosello-Trampont ${ }^{1,4}$, JM Kinchen ${ }^{1,2,4}$, E Brugnera ${ }^{1,3}$, LB Haney ${ }^{1}$, MO Hengartner ${ }^{2}$ and KS Ravichandran ${ }^{\star, 1}$
}

Removal of apoptotic cells is a dynamic process coordinated by ligands on apoptotic cells, and receptors and other signaling proteins on the phagocyte. One of the fundamental challenges is to understand how different phagocyte proteins form specific and functional complexes to orchestrate the recognition/removal of apoptotic cells. One evolutionarily conserved pathway involves the proteins cell death abnormal (CED)-2/chicken tumor virus no. 10 (CT10) regulator of kinase (Crk)II, CED-5/180 kDa protein downstream of chicken tumor virus no. 10 (Crk) (Dock180), CED-12/engulfment and migration (ELMO) and MIG-2/RhoG, leading to activation of the small GTPase CED-10/Rac and cytoskeletal remodeling to promote corpse uptake. Although the role of ELMO : Dock180 in regulating Rac activation has been well defined, the function of CED-2/Crkll in this complex is less well understood. Here, using functional studies in cell lines, we observe that a direct interaction between Crkll and Dock180 is not required for efficient removal of apoptotic cells. Similarly, mutants of CED-5 lacking the CED-2 interaction motifs could rescue engulfment and migration defects in CED-5 deficient worms. Mutants of Crkll and Dock180 that could not biochemically interact could colocalize in membrane ruffles. Finally, we identify MIG-2/RhoG (which functions upstream of Dock180:ELMO) as a possible point of crosstalk between these two signaling modules. Taken together, these data suggest that Dock180/ELMO and Crkll act as two evolutionarily conserved signaling submodules that coordinately regulate engulfment.

Cell Death and Differentiation (2007) 14, 963-972. doi:10.1038/sj.cdd.4402094; published online 16 February 2007

Apoptotic cells are generated by diverse physiological processes, ranging from the elimination of damaged (or precancerous) cells to deletion of cells during developmental morphogenesis; $;^{1,2}$ the culmination of the apoptotic program is the phagocytosis (or engulfment) of the apoptotic cell. In mammals, prompt removal of apoptotic cells is required to prevent the release of potential self-antigens and onset of autoimmune-like syndromes. $^{3-5}$

Removal of the apoptotic cell can be divided into several mechanistic steps: first, dying cells expose 'eat-me' signals, which are recognized by receptors on phagocytic cells. ${ }^{6}$ These signals, both positive (such as phosphatidylserine and MFG-E8) ${ }^{2}$ and negative (such as CD31 and CD47), are rapidly modified following the onset of apoptosis, facilitating removal of the apoptotic cell. A number of receptors have been identified that either directly or indirectly facilitate apoptotic cell recognition; however, signaling following corpse recognition is less well characterized.

Recognition of the apoptotic cell by the phagocyte leads to the formation of an actin-rich phagocytic cup, followed by the internalization of the target. ${ }^{7}$ Genetic studies in the nematode
Caenorhabditis elegans (C. elegans) have led to the identification of eight genes that play a partially redundant role in removal of apoptotic cells. ${ }^{2}$ Of these, cell death abnormal (CED)-2/chicken tumor virus no. 10 (CT10) regulator of kinase (Crk)II, CED-5/180 kDa protein downstream of Crk (Dock180), CED-10/Rac, CED-12/engulfment and migration (ELMO), MIG-2/RhoG and UNC-73/Trio have been subsequently shown to function together in corpse removal in the mammalian system.

The mammalian homologue of CED-5, Dock180, is a prototype member of a protein superfamily that was recently identified as novel guanine nucleotide exchange factors (GEFs) for small GTPases. Dock180 forms a basal complex with the CED-12 homologue, ELMO1, and together this complex functions as an unconventional two-part GEF specific for Rac; 8 overexpression of ELMO with Dock180 leads to increased Rac-guanine triphosphate (GTP) levels within the cell. ${ }^{8}$ As with other GTPases, Rac functions as a binary switch by cycling between an inactive guanine diphosphate (GDP)-bound and an active GTP-bound form. Dock180 and its homologues contain a Docker domain that

\footnotetext{
${ }^{1}$ Beirne Carter Center for Immunology Research and the Department of Microbiology, University of Virginia, Charlottesville, Virginia, USA; ${ }^{2}$ Institute of Molecular Biology, University of Zurich, Zurich, Switzerland and ${ }^{3}$ Institute of Veterinary Pathology, Vetsuisse Faculty, University of Zurich, Zurich, Switzerland

*Corresponding author: KS Ravichandran, Carter Immunology Center, University of Virginia, MR4- Rm4072D, Box 801386, Lane Road, Charlottesville, Virginia 22908, USA. Tel: + 1434243 6093; Fax: +1 434924 1221; E-mail: Ravi@ virginia.edu

${ }^{4}$ These authors contributed equally to this work.

Keywords: phagocytosis; engulfment; apoptotic cells ; Crkll; Dock 180; Rac

Abbreviations: CED, cell death abnormal; $C$. elegans, Caenorhabditis elegans; CRIB, cdc42/Rac interactive binding domain; Crk, chicken tumor virus no. 10 (CT10) regulator of kinase; CZH1, CDM-Zizimin Homology 1; Dock180, $180 \mathrm{kDa}$ protein downstream of Crk; DHR1, Dock homology region 1; DTC, distal tip cell; ELMO, engulfment and migration; GDP, guanine diphosphate; GTP, guanine triphosphate; GEF, guanine nucleotide exchange factor; MFI, mean fluorescence index; PH domain, Plextrin Homology domain; Ptdlns(3,4,5) $\mathrm{P}_{3}$, phosphatidylinositol 3,4,5-triphosphate; PtdSer, PhosphatidylSerine; SH2, Src Homology 2; SH3, Src Homology 3; siRNA, short interfering RNA

Received 04.9.06; revised 28.11.06; accepted 28.11.06; Edited by M Piacentini; published online 16.2.07
} 
can interact directly with nucleotide-free Rac and mediate Rac GDP/GTP exchange in vitro; 8,9 Rac activation mediated via the Dock180: ELMO complex has been linked to cytoskeletal changes during engulfment and cell migration. ${ }^{10,11}$ Two additional players, MIG-2/RHOG and UNC-73/Trio, play a role upstream of ELMO : Dock180. ${ }^{12,13}$ The binding of ELMO to activated GTP-bound RhoG may serve as one mechanism for recruitment of the ELMO:Dock180 complex to the membrane, leading to Rac activation.

Although the role of ELMO : Dock180 has been the subject of intense research, the function of CED-2/Crkll in this complex is less well understood. Crkll, Dock180 and ELMO have been shown to interact in a ternary complex, ${ }^{10}$ but the contribution or requirement for Crkll is poorly defined. Dock180 was initially cloned based on its interaction with Crkll, ${ }^{14}$ suggesting that CED-2/Crkll serves as an adapter protein that helps to recruit Dock180 to the cell membrane during engulfment, leading to Dock180: ELMO-mediated Rac activation. ${ }^{10}$ However, the requirement for Crkll during mammalian engulfment, and how Crkll regulates Dock180: ELMO function are not understood. Here, we address the importance of Crkll and its association with Dock180 in promoting engulfment. We find that contrary to previously proposed models, interaction of Crkll with Dock180 is not required for engulfment based on genetic studies in worms as well as biochemical and functional studies in mammalian cells. Moreover, our work identifies two submodules within this Crkll/Dock180/ELMO signaling pathway that work in concert to promote maximal engulfment.

\section{Results}

CED-2 binding sites on CED-5 are not required for engulfment and cell migration in vivo. The ced-2, ced-5, ced-10 and ced-12 genes, which code for mammalian homologues of Crkll, Dock180, Rac and ELMO, respectively, are required in the nematode $C$. elegans for efficient removal of apoptotic cell corpses. ${ }^{10,15-18}$ Previous studies have shown that CED-2 and CED-5 physically interact; ${ }^{15}$ however, the importance of the CED-2:CED-5 interaction in regulating phagocytosis has not been addressed. To examine the in vivo relevance of CED2:CED-5 interaction, we generated a mutant of CED-5 (denoted CED-5(1-1715)) lacking the motifs required for binding CED-2 (see Figure 1a). This mutant has an intact a

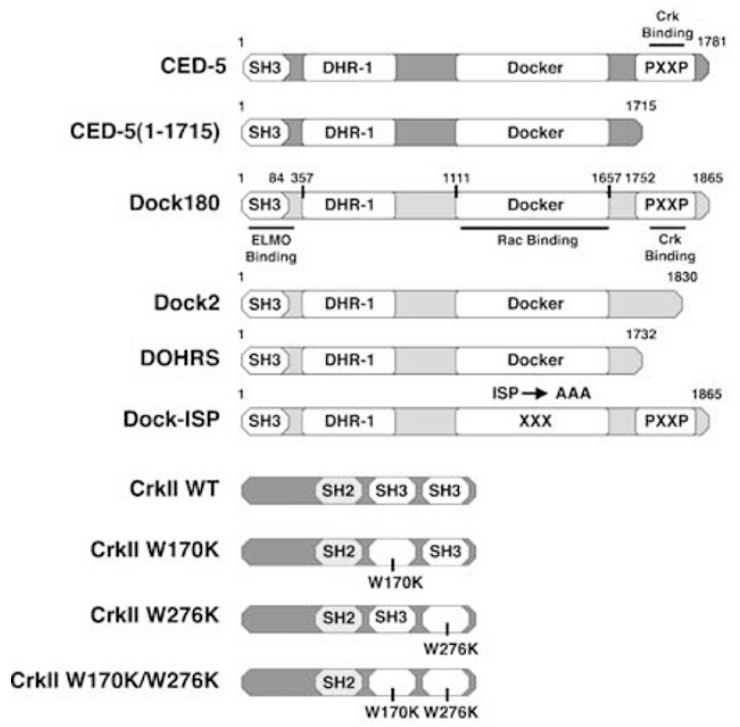

b

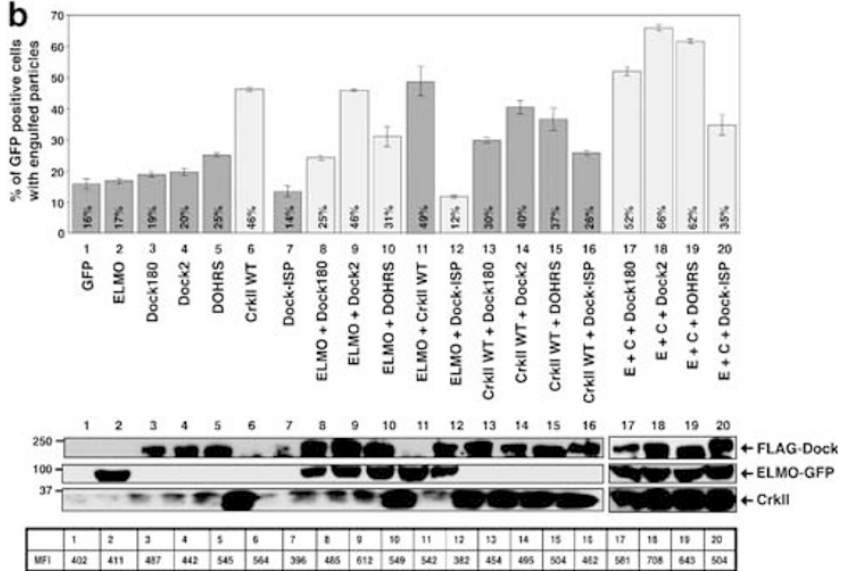

C

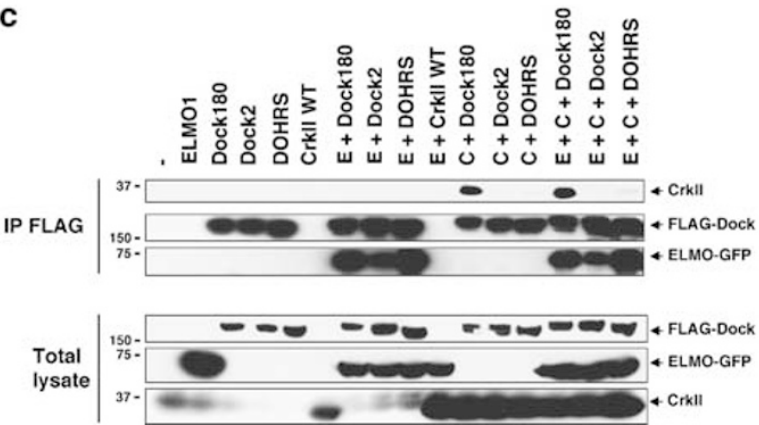

Figure 1 Direct interaction of Crkll with Dock180 is not required for Crkll to cooperate with the Dock180: ELMO proteins in promoting phagocytosis. (a) Schematic representation of various constructs used in this study. The DHR1 (named also CZH1), Docker (termed also DHR-2 or CZH2), SH2, SH3 and PXXP domains are displayed. (b) LR73 cells were transiently transfected in triplicate with the indicated plasmids. Phagocytosis by GFP-positive cells was assessed by two-color flow cytometry and the percentage of GFP-positive cells that had ingested surrogate apoptotic targets (red fluorescent) is indicated for each condition as described previously. ${ }^{24}$ Mean of fluorescence intensity (MFI), a measure of the number of particles per engulfing cell (in arbitrary MFI units) is indicated in the table below. The expression of the transfected proteins was confirmed by immunoblotting of total lysates as indicated. Note that the blot of Crkll shows both endogenous and overexpressed Crkll proteins. (c) LR73 cells were transiently transfected with the indicated proteins, lysed and immunoprecipitated using anti-Flag conjugated agarose beads and assessed by immunoblotting for the coprecipitating proteins. E, ELMO1; C, Crkll 
Table 1 (a) CED-5 mutant lacking CED-2 binding motif can mediate corpse removal in CED-5 null worms, (b) CED-5 mutant lacking the CED-2 binding motif can rescue DTC migration in CED-5- null worms, (c) two Crkll SH3 domains function redundantly in rescue of DTC migration in CED-2 mutants

\begin{tabular}{|c|c|c|}
\hline Genotype & Corpse Number & $\mathbf{n}$ \\
\hline \multicolumn{3}{|c|}{ (a) CED-5 mutant lacking CED-2 binding motif can mediate corpse removal in CED-5 null worms } \\
\hline Wild type & 0 & 10 \\
\hline opEx756 [P eft-3::ced-5(WT)] & 0 & 20 \\
\hline opEx772 [P eft-3::ced-5(1-1715)] & 0 & 20 \\
\hline 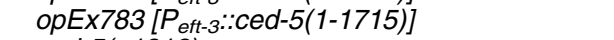 & 0 & 20 \\
\hline ced-5(n1812) & $20.3 \pm 6.9$ & 20 \\
\hline ced-5(n1812); opEx756 [P eft-3::ced-5(WT)] & $4.4 \pm 3.1$ & 10 \\
\hline ced-5(n1812); opEx772 [P eft-3::ced-5(1-1715)] & $1.7 \pm 2.8$ & 10 \\
\hline ced-5(n1812);opEx783 [P eft-3::ced-5(1-1715)] & $3.3 \pm 3.1$ & 10 \\
\hline ced-12(k149) & $16.8 \pm 5.0$ & 20 \\
\hline ced-12(k149); opEx756 [P eft-3::ced-5(WT)] & $25.2 \pm 7.6$ & 10 \\
\hline ced-12(k149); opEx772 [P eft-3::ced-5(1-1715)] & $18.8 \pm 5.3$ & 10 \\
\hline ced-12(k149);opEx783 [P eft-3::ced-5(1-1715)] & $18.3 \pm 7.9$ & 21 \\
\hline \multicolumn{3}{|c|}{ (b) CED-5 mutant lacking the CED-2 binding motif can rescue DTC migration in CED-5- null worms } \\
\hline Genotype & Migration (\%) & $\mathbf{n}$ \\
\hline wild type & 0 & 204 \\
\hline opEx732 [P $P_{\text {eft-3::ced-5(WT)] }}$ & 0.06 & 326 \\
\hline 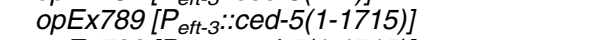 & 3 & 304 \\
\hline opEx790 $\left[P_{\text {eft-3: }:: c e d-5(1-1715)]}\right.$ & 3 & 326 \\
\hline opEx790 [P $P_{\text {eft-3: }:: c e d-5(1-1715)]}$ & 1 & 312 \\
\hline ced-5(n1812) & 35 & 314 \\
\hline ced-5(n1812); opEx732 [P & 5 & 173 \\
\hline ced-5(n1812); opEx789 [P eft-3::ced-5(1-1715)] & 12 & 304 \\
\hline ced-5(n1812); opEx790 [P eft-3::ced-5(1-1715)] & 10 & 314 \\
\hline ced-5(n1812); opEx794 [P eft-3::ced-5(1-1715)] & 12 & 314 \\
\hline ced-12(k149) & 36 & 305 \\
\hline 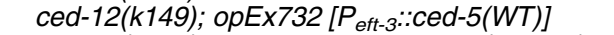 & 29 & 306 \\
\hline ced-12(k149); opEx789 [P eft-3::ced-5(1-1715)] & 41 & 312 \\
\hline 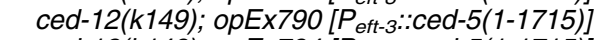 & 39 & 310 \\
\hline ced-12(k149); opEx794 [P eft-3::ced-5(1-1715)] & 32 & 313 \\
\hline \multicolumn{3}{|c|}{ (c) Two Crkll SH3 domains function redundantly in rescue of DTC migration in CED-2 mutants } \\
\hline Genotype & Migration (\%) & $\mathbf{n}$ \\
\hline wild type & 0 & 204 \\
\hline ced-2(e1752) & 31 & 307 \\
\hline ced-2(e1752); opEx891 [P eft-3::ced-2(WT)] & 6 & 312 \\
\hline ced-2(e1752); opEx897 [P eft-3::crkll(WT)] & 14 & 294 \\
\hline ced-2(e1752); opEx898 [P eft-3::crkll(W170K)] & 15 & 313 \\
\hline 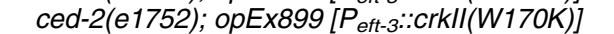 & 9 & 318 \\
\hline ced-2(e1752); opEx929 [P eft-3::crkll(W170K)] & 10 & 310 \\
\hline ced-2(e1752); opEx946 [P $P_{\text {eft-3::crkll }(W 276 K)]}$ & 9 & 308 \\
\hline ced-2(e1752); opEx947 [P eft-3::crkll(W276K)] & 18 & 308 \\
\hline 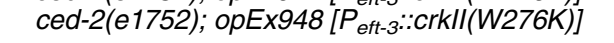 & 11 & 300 \\
\hline
\end{tabular}

Transgenic worms were scored for the presence of apoptotic corpses in the L1 head (shown \pm S.D.). $n$, number of worms, or scored DTC migration defects in the early adult (b, c).

CED-12/ELMO binding region as well as an intact Docker domain required for CED-10/Rac activation, allowing us to specifically test the requirement of CED-5 binding to CED-2 for the function of this mutant. Surprisingly, CED-5(1-1715) was still able to efficiently rescue the engulfment defect of ced-5 mutant worms, comparable to wild-type CED-5 (Table 1a). Importantly, the CED-5(1-1715) mutant did not rescue the engulfment defect of ced-12 mutant worms, suggesting that CED-5(1-1715) is not an activating mutation (Table 1a). Thus, the CED-2 biding motif in CED-5 is not required for efficient clearance of apoptotic bodies in vivo.

In addition to defects in engulfment, worms deficient in ced2, ced-5, ced-10 and ced-12 also present defects in cell migration. ${ }^{19,20}$ To address whether CED-2 : CED- 5 interaction is required for cell migration, we assayed migration of the gonadal distal tip cells (DTCs), which define the shape of the adult hermaphrodite gonad. ${ }^{12}$ Interestingly, expression of CED-5(1-1715) in CED-5-deficient worms was able to rescue
DTC migration as efficiently as the wild-type CED-5 (Table 1b). These data suggested that the direct interaction of CED-2 and CED-5 is not required for efficient engulfment or DTC migration in $C$. elegans.

Dock180 lacking Crkll-binding sites can promote engulfment in mammalian cells. Previous work has shown that Dock180 and ELMO proteins promote phagocytosis in mammalian cells by a Rac-dependent signaling pathway. ${ }^{8,10}$ Crkll is able to cooperate with ELMO:Dock180 to further enhance uptake of apoptotic targets. ${ }^{10}$ It has been assumed that Crkll participates in phagocytosis by binding to Dock180, and that Crkll, either directly or indirectly, might recruit the Dock180:ELMO complex to the membrane to mediate cytoskeletal rearrangement during engulfment. ${ }^{10}$ To test this notion in a functional assay, we used a mutant of Dock180 (DOHRS) that can bind ELMO, but not Crkll (Figure 1a and $c$ and 

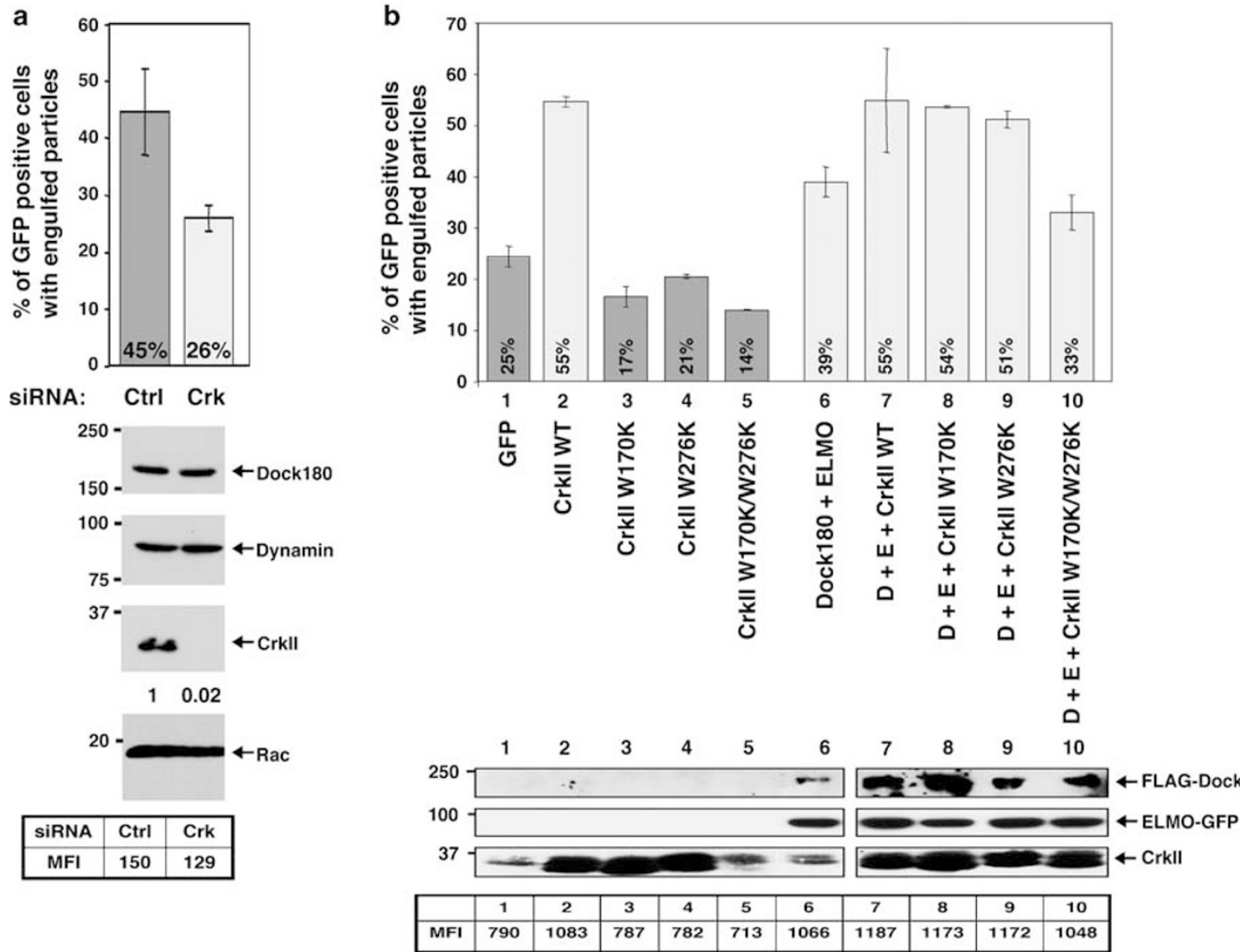

Figure 2 Crkll is required for phagocytosis. (a) NIH/3T3 cells were transfected with Crk siRNA and phagocytic ability was determined as in Figure 1. Transfection efficiency was close to $95 \%$ for $\mathrm{NIH} / 3 \mathrm{~T} 3$ cells (data not shown). (b) LR73 cells were transiently transfected with the indicated plasmids. The ability of transfected cells to phagocytosis surrogate apoptotic targets (red fluorescent) was analyzed by two-color cytometry as described in Figure 1b. The efficiency of uptake of the apoptotic targets by the transfected cells is indicated by their MFI in the table below. E, ELMO1; D, Dock180

Supplementary Figure 1). ${ }^{8,21-23}$ Dock2, a member of Dock180 superfamily, ${ }^{9}$ can bind ELMO, but lacks the Crkll interaction motif (Figure 1a and c). When coexpressed with ELMO1, both Dock2 and DOHRS proteins were able to enhance phagocytosis, similar to Dock180 (Figure 1b, bars 8, 9 and 10 compared to 2, 3, 4 and 5), whereas a catalytically inactive mutant of Dock180 (Dock-ISP) ${ }^{8}$ that does not stimulate Rac-GTP loading failed to promote phagocytosis (Figure 1b, bar 12 compared to 8). Interestingly, Crkll was still able to further enhance phagocytosis mediated by ELMO:Dock2 or ELMO:DOHRS (Figure 1b, bars 17, 18 and 19 compared to bars 8, 9 and 10). One possible explanation for this observation could be that although Crkll fails to bind Dock2 or DOHRS proteins, Crkll might form a higher order complex in the presence of ELMO. However, we could not detect Crkll associated with either Dock2 or DOHRS in the presence or absence of ELMO (Figure 1c and Supplementary Figure S1). Together, these data suggested that for Dock180 to function in phagocytosis, a direct interaction with Crkll is not required.

Crkll is required for efficient removal of apoptotic targets. Our results suggest that Crkll interaction was dispensable for enhancement of phagocytosis upon
ELMO:Dock180 overexpression, which highlighted the possibility that Dock180 function may not require Crkll. Previous studies have shown that, when overexpressed, Crkll robustly promotes engulfment of apoptotic targets; ${ }^{24}$ however, loss-of-function assays have not been conducted to determine a requirement for Crkll in mammalian engulfment. Short interfering RNA (siRNA)-mediated knockdown of Crkll in NIH/3T3 mouse fibroblasts significantly decreased the uptake of targets (Figure 2a), suggesting that Crkll-mediated signaling plays a role in efficient uptake of apoptotic targets. This result is consistent with studies in the nematode where mutations in the Crkll homologue CED-2 results in persistent cell corpses. ${ }^{15}$

Binding to dock180 is dispensable for Crkll to cooperate with the Dock180/ELMO during engulfment. Next, we asked how mutants of Crkll that fail to bind Dock180 would cooperate with the Dock180:ELMO complex to promote engulfment. Crkll is an adapter protein composed of one $\mathrm{N}$-terminal Src Homology 2 (SH2) domain and two C-terminal Src Homology 3 (SH3) domains (Figure 1a). ${ }^{25}$ The first $\mathrm{SH} 3$ domain binds Dock180; a mutation within this $\mathrm{SH} 3$ domain (W170K) abrogates Crkll binding to Dock180 (Figure 3b). ${ }^{26}$ Although expression of CrkII ${ }^{\mathrm{WT}}$ robustly promoted uptake, 


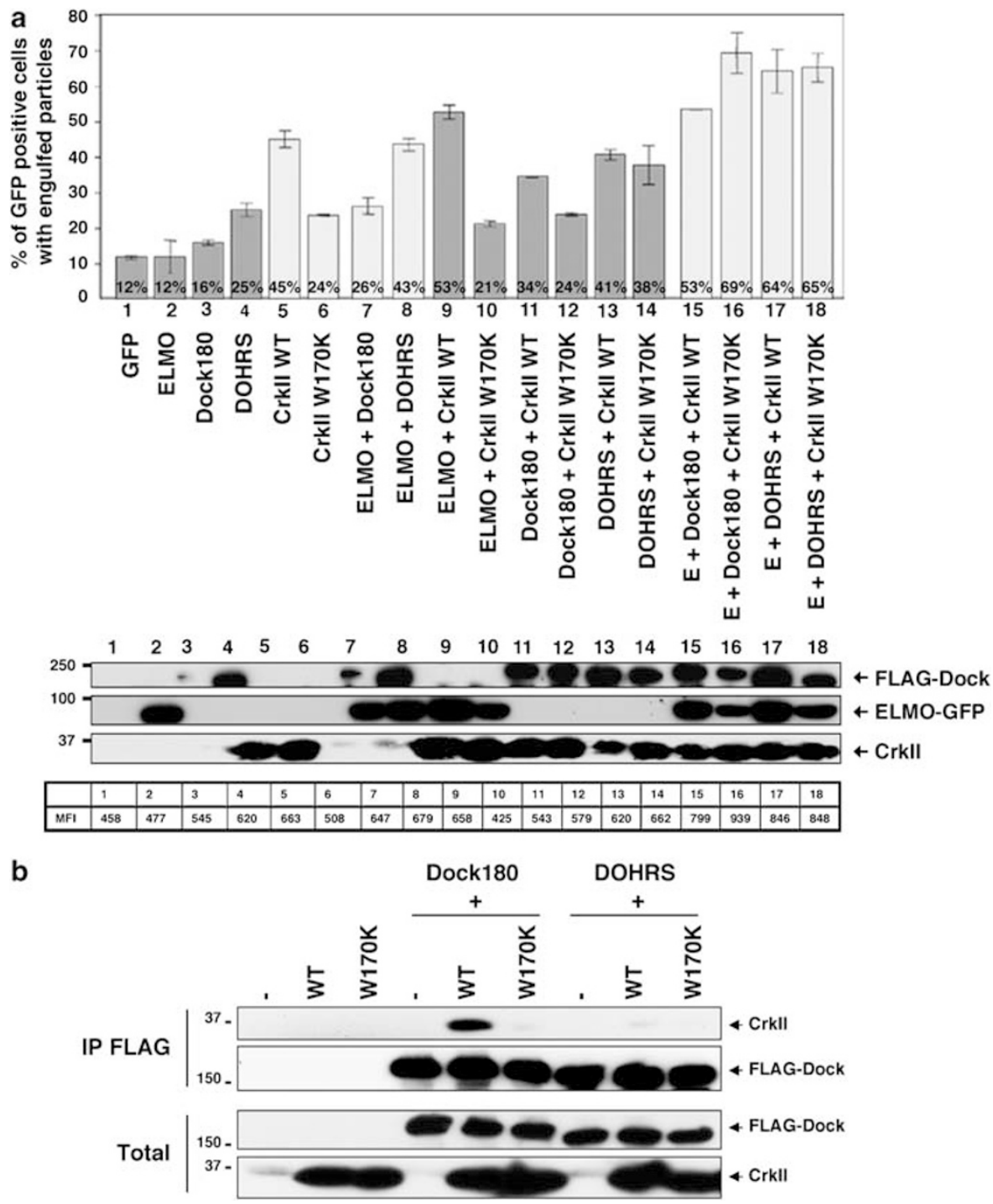

Figure 3 Dock180: Crkll interaction is not required for the two proteins to cooperate during mammalian engulfment. (a) LR73 cells were transfected with the indicated plasmids, and the phagocytosis assay was performed as described in Figure 1b. (b) LR73 cells were transiently transfected, lysed and protein immunoprecipitated using antiFlag conjugated to agarose beads. The ELMO1 blot indicates endogenous ELMO proteins associated to Dock180 or DOHRS. E, ELMO1

CrkllW170K was unable to promote phagocytosis ${ }^{24}$ (Figure 2b, bar 3 compared to 2; and Figure 3a, bar 6 compared to 5). However, this CrkII ${ }^{\mathrm{W} 170 \mathrm{~K}}$ mutant was as efficient as CrkII ${ }^{\mathrm{WT}}$ in promoting uptake when coexpressed with Dock180: ELMO (Figure 2b, bar 8 compared to bars 7 and 6; Figure 3a, bar 16 compared to bars 15 and 7). Further, coexpression of DOHRS and CrkII ${ }^{\mathrm{W} 170 \mathrm{~K}}$, which removes the Crkll: Dock180 interaction motifs in both proteins, was still able to promote phagocytosis (Figure $3 a$, bar 18 compared to bars 15, 16 and 17). To rule out any unexpected interactions between CrkII ${ }^{\mathrm{W} 170 \mathrm{~K}}$ and the DOHRS mutant, we confirmed by immunoprecipitation that CrkII ${ }^{\mathrm{W} 170 \mathrm{~K}}$ fails to bind the DOHRS mutant of Dock180 (Figure 3b and Supplementary Figure S2). These data showed that the Dock180:Crkll interaction is not required for the two proteins to cooperate during mammalian engulfment.

The ability of the CrklI ${ }^{\mathrm{W} 170 \mathrm{~K}}$ mutant to cooperate with Dock180: ELMO in promoting maximal engulfment prompted us to examine the importance of the second SH3 domain of Crkll. Although this domain has no known binding partners during engulfment, based on structurally conserved residues among SH3 domains, a mutation (W276K) has been described which should disrupt this motif. ${ }^{27,28}$ This CrkII ${ }^{\text {W276K }}$ mutant was defective in promoting uptake when individually expressed (Figure $2 \mathrm{~b}$, bar 4 compared to 1 ), but was still able to cooperate with Dock180 (comparable to the wild-type Crkll construct) when coexpressed (Figure $2 \mathrm{~b}$, bar 9, compared to bars 6 and 7). However, Crkll with mutations in both $\mathrm{SH} 3$ domains (W170K/W276K) failed to cooperate with Dock180 and ELMO to promote phagocytosis when coexpressed (Figure $2 \mathrm{~b}$, bar 10 versus bar 6 ), suggesting that the two $\mathrm{SH} 3$ domains of Crkll might function redundantly.

To further address the relevance of the Crkll SH3 domains in vivo, we performed rescue studies in CED-2 deficient worms. Consistent with our previous observations, CrkII ${ }^{\mathrm{W} 170 \mathrm{~K}}$ and CrkII ${ }^{\text {W276K }}$ mutants were able to rescue DTC migration in 
a

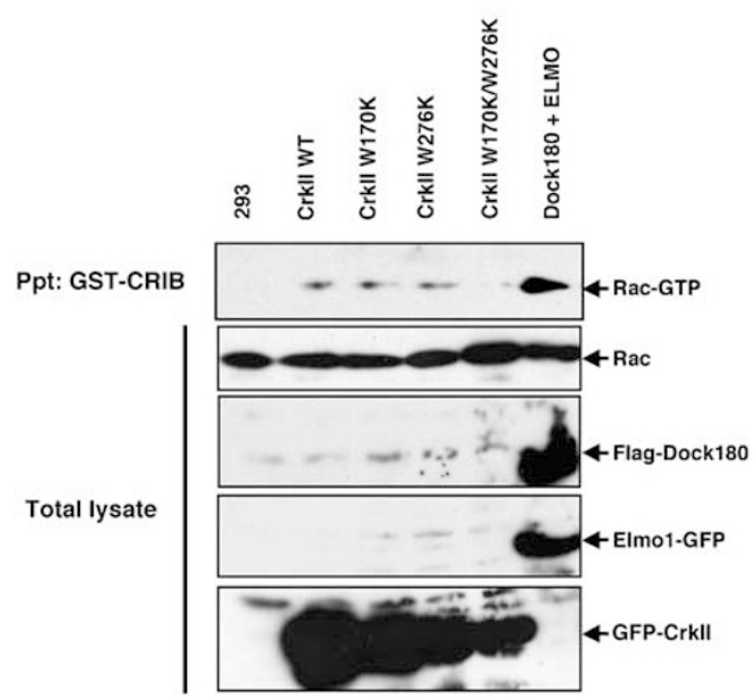

b

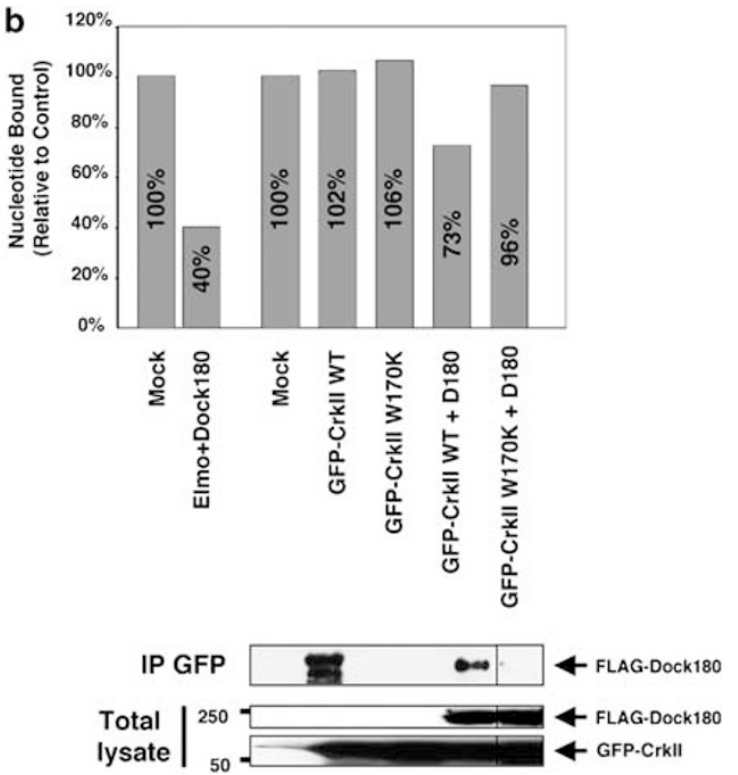

Figure 4 Overexpression of Crkll does not enhance Rac activation in vitro or in vivo. (a) 293T cells were transiently transfected as indicated, lysed, and GTP-bound Rac was precipitated using bacterially purified GST-CRIB domain, and assessed by immunoblotting for Rac. Expression of transfected proteins was determined by immunoblotting of total cell lysates. (b) GFP-CrkII ${ }^{\mathrm{WT}}$ GFP-CrkII ${ }^{\mathrm{W} 170 \mathrm{~K}}$ and Dock180 were expressed in $293 \mathrm{~T}$ cells as indicated. Cell lysates were precipitated using anti-GFP-conjugated beads. His-Dock180 coexpressed with Flag-ELMO1, and immunoprecipitated by anti-Flag conjugated beads was used as positive control. The radioactivity bound to Rac in the mocktransfected 293T cell condition was set as $100 \%$ and the loss of radioactivity indicates the presence of Rac-GEF activity in the precipitated samples. The expression of the transfected proteins and the presence of Flag-Dock180 in the different precipitates were detected by immunoblotting. D, Dock180

CED-2 deficient worms as efficiently as CrklI ${ }^{\mathrm{WT}}$ (Table 1c). This confirmed that both in the context of cell lines and in the whole organism, a direct interaction with CED-5/Dock180 is dispensable for the function of Crkll.

Crkll does not directly regulate Rac directly. The above data suggest that although either of the $\mathrm{SH} 3$ domains of Crkll can cooperate with Dock180:ELMO complex during engulfment, a physical interaction between Crkll and Dock180 (or ELMO) is not required. Only the first SH3 domain of Crkll can bind Dock180; in functional assays, the first or the second $\mathrm{SH} 3$ domain could cooperate with Dock180: ELMO to promote engulfment. This implied that interaction of Crkll with additional proteins might serve to compensate for the loss of the Crkll: Dock180 interaction. Crkll mediated uptake is inhibited by dominant-negative $\mathrm{Rac}^{\mathrm{N} 17,24}$ it is thus possible that Crkll might associate with another Rac-GEF (in addition to, or instead of Dock180) to promote uptake. However, transient overexpression of Crkll alone or the mutants of Crkll had only a minimal effect on endogenous Rac-GTP levels in cell (Figure 4a). In comparison, coexpression of Dock180: ELMO significantly increased the Rac-GTP in cells; ${ }^{8,10,29}$ co-expression of Crkll with ELMO:Dock180 did not consistently augment the increase in cellular Rac-GTP induced by the ELMO: Dock180 complex (Supplementary Figure 3).

We next examined whether an additional Rac-GEF activity could be co-immunoprecipitated with Crkll. CrkII ${ }^{\mathrm{WT}}$ coexpressed with Dock180 had detectable association with a Rac-GEF activity; however, CrkII ${ }^{\mathrm{W} 170 \mathrm{~K}}$ lost its ability to precipitate this Rac-GEF activity, which correlated with an inability to coprecipitate Dock180 (Figure 4b). Taken together, these data suggested that Crkll-mediated regulation of Rac depends on Dock180: ELMO proteins and is unlikely to involve another Rac-GEF. Moreover, our data suggest that although the second $\mathrm{SH} 3$ domain of Crkll is able to substitute for the loss of interaction with Dock180 via the first SH3 domain, this is not accomplished by mediating a direct or indirect interaction with ELMO : Dock180, revealing a potentially Dock180-independent signaling event. Consistent with this interpretation, overexpression of a catalytically inactive mutant of Dock180, Dock-ISP, was able to only partially inhibit CrkII ${ }^{\mathrm{WT}}$ induced uptake (Figure 1b, compare bars 6 and 16).

Crkll colocalizes with Dock180 and ELMO proteins independent of direct physical interaction. It has been postulated that the role of Crkll might be to mediate localization of Dock180: ELMO to the cell membrane, ${ }^{10}$ potentially serving as an adaptor protein between an unidentified transmembrane receptor and Dock180: ELMO mediated Rac activation. We find that Crkll mutants that fail to bind Dock180 (and vice versa) functionally cooperate during engulfment. This suggests that Crkll and Dock180 mutants that may not physically bind each other might still colocalize in the cell. Indeed, both Dock180 and DOHRS mutants colocalized with Crkll in membrane ruffles, whereas overexpressed GFP was excluded from these structures (Figure 5 and Supplementary Figure 4). Similarly, when CrkII ${ }^{\text {W170K }}$ mutant was coexpressed with ELMO : Dock180 or with ELMO:DOHRS, all three proteins colocalized at the membrane ruffles (Figure 5). These data suggest that membrane localization of Crkll or Dock180: ELMO is not 

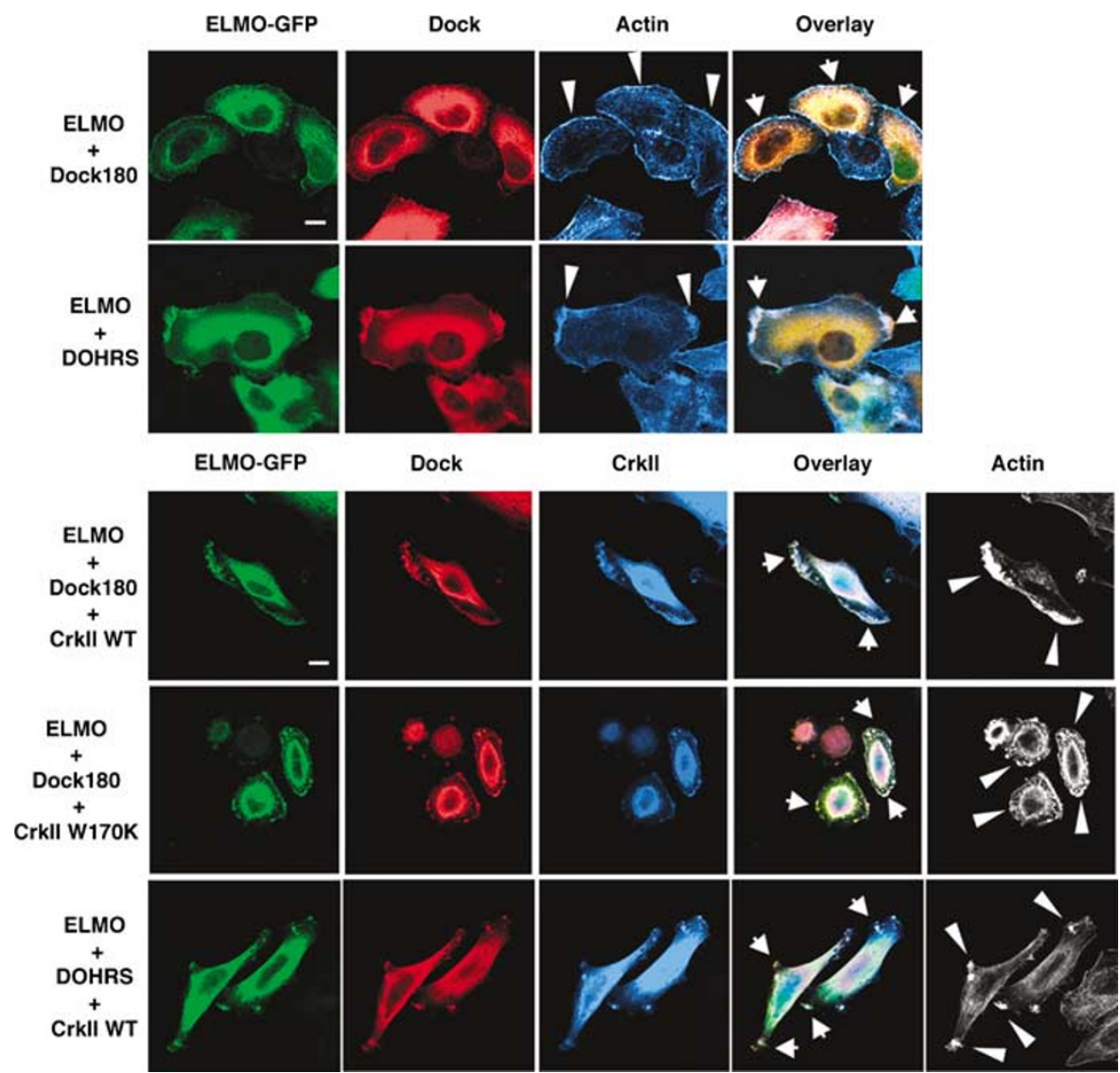

Figure 5 Dock180 and ELMO do not require interaction with Crkll for localization to membrane ruffles. HeLa cells were transfected with the indicated plasmids and the colocalization of Crkll, CrkII ${ }^{\mathrm{W} 170 \mathrm{~K}}$, Dock180, DOHRS, ELMO and polymerized actin (as measured by phalloidin staining) were assessed by confocal microscopy. Arrowheads indicate the colocalization of Crkll, Dock180 and ELMO proteins in membrane ruffles

dependent on the ability of these proteins to interact physically.

Other potential mechanisms may mediate localization of these proteins; the Dock homology region 1 (DHR1) domain within Dock180, which binds to phosphatidylinositol 3,4,5triphosphate $\left(P t d l n s(3,4,5) P_{3}\right)$, or additional sequences within the C-terminus of Dock180 may allow for direct membrane recruitment. ${ }^{21,30}$ However, in our experiments to date, inhibition of Ptdlns $(3,4,5) \mathrm{P}_{3}$ fails to inhibit the uptake mediated by Dock180: ELMO proteins (data not shown), suggesting that Ptdlns $(3,4,5) \mathrm{P}_{3}$ binding is not the primary determinant of Dock180 recruitment during engulfment.

RhoG as a possible intersection point between Crkll and the Dock180/ELMO signaling modules. The small GTPase RhoG has recently been suggested to mediate ELMO : Dock180 complex recruitment to the membrane, in turn potentially regulating Rac activation during engulfment. ${ }^{12,13}$ We next asked whether RhoG might serve as a link between the Crkll and the Dock180:ELMO submodules. When we coexpressed a dominant-negative form of RhoG (RhoG ${ }^{T 17 N}$ ) with Crkll, it was able to partially inhibit Crkll-mediated phagocytosis (Figure 6, compare bars 8-2). Basal phagocytosis was also slightly decreased (Figure 6, compare bar 1-5), but we believe the inhibition of Crkll by RhoG ${ }^{N 17}$ is specific and not a general effect of decreased phagocytic potential (see Discussion). This suggested then that RhoG might provide at least one potential intersection point between Crkll and Dock180: ELMO signaling, consistent with earlier worm studies where the number of unengulfed corpses in CED-2-deficient animals was enhanced by the concurrent loss of the RhoG homologue MIG-2. ${ }^{12,20}$ However, the partial inhibition of Crkll-mediated engulfment by dominant-negative RhoG could suggest that Crkll may also link to the Dock180/ ELMO module independent of RhoG, or that Crkll also mediates RhoG-independent signals. We hypothesize that one or both of the $\mathrm{SH} 3$ domains of Crkll might link Crkll-mediated signaling to RhoG-dependent or RhoGindependent pathways.

\section{Discussion}

In recent years, a number of proteins have been described that mediate recognition of the apoptotic cell corpse. However, one of the fundamental challenges of the field is to understand how different players form specific and functional complexes to orchestrate the recognition and removal of apoptotic cells. CED-2, CED-5, CED-10 and CED-12 function in the same genetic pathway in the nematode; ${ }^{2}$ however, the mechanism by which these proteins cooperate to promote phagocytosis is poorly understood. Dock180 was initially 


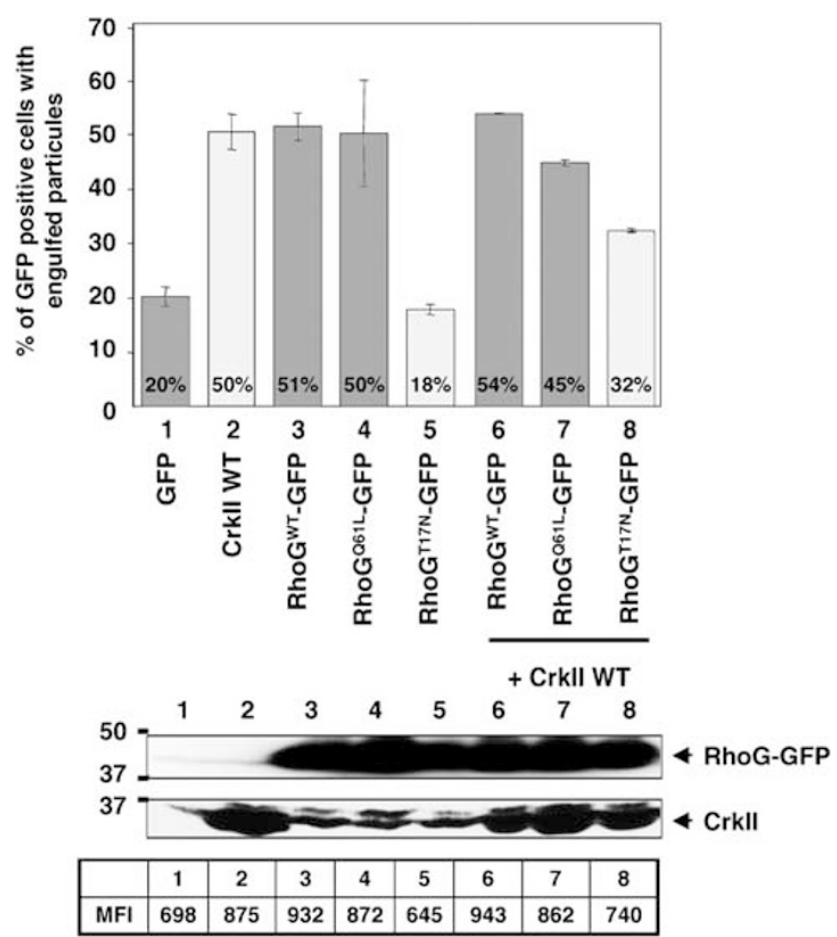

Figure 6 Crkll mediates RhoG-independent and RhoG-dependent signals. LR73 cells were transfected with the indicated plasmids, and the phagocytosis assay was performed as described in Figure $1 \mathrm{~b}$. Note that the blot for Crkll shows both the endogenous and overexpressed Crkll

identified as a Crkll interacting protein; ${ }^{14}$ it has been widely assumed that Crkll and Dock180 form a physical complex required for their function during engulfment. We present genetic, biochemical and functional evidence that direct physical association between Crkll and Dock180 is not essential for these proteins to function during engulfment and that Crkll and Dock180-mediated signaling may represent two submodules within the same pathway.

With the identification of the catalytic role of the ELMO: Dock180 complex, it has been unclear what precise role Crkll might play in regulating Dock180 function. Although many models propose Crkll as a means for recruiting Dock180 to the membrane, membrane targeting sequences within Dock $180^{21}$ and a potential Plextrin Homology domain in ELMO $^{18}$ have been suggested to provide efficient targeting sequences. Further, interaction of ELMO:Dock180 with proteins such as $\mathrm{RhoG}^{12,13}$ may also provide localization cues. The data presented here demonstrate that physical interaction between Dock 180 and Crkll is dispensable for their function in phagocytosis. This is the case both in mammalian cell lines and in the whole organism, where we used rescue experiments in $C$. elegans strains deficient in the Crkll and Dock180 homologues CED-2 and CED-5. Moreover, colocalization of CrkII ${ }^{\mathrm{W} 170 \mathrm{~K}}$ mutants with a Dock180 mutant (DOHRS) lacking the Crkll interaction sites suggest that colocalization alone does not reflect a physical association and that recruitment of these proteins is likely independent. Alternately, an as-yet unidentified protein may bridge these proteins in vivo, effectively tethering their activities close enough to allow coordinate function.
This has led us to reconsider the function of Crkll during phagocytosis. One possibility is that, upon receptor activation, these proteins are enriched at a given area of the cell surface resulting in efficient Rac activation and particle uptake (rather than a simple direct role of Crkll in recruitment of Dock180 from the cytoplasm to the membrane). The nematode protein CED-1, which is believed to function as a receptor for the apoptotic, has been suggested to function in just this manner. ${ }^{31}$ Thus, we would like to hypothesize that perhaps Crkll would function to mediate enrichment of proteins within the phagocytic cup, where Crkll would synergize with the Dock180 : ELMO complex in regulating Rac to further promote the phagocytic process. We have attempted to detect such a recruitment of endogenous Crkll to the phagocytic cup, but were unable to obtain an antibody that convincingly stained endogenous protein; overexpression of the proteins did not yield distinct localization patterns in the phagosome (data not shown). Alternately, Crkll may serve no role in localization, and instead function as a bridging molecule, uniting divergent pathways to couple recognition of the apoptotic cell to the actin polymerization machinery.

Although direct interaction between Dock180 and Crkll is not required for function in corpse removal, we show using siRNA-mediated knockdown that Crkll is required for efficient phagocytosis, consistent with studies in the nematode. ${ }^{15}$ Our data show that even though Crkll promotes strong engulfment in the in vitro assays, Crkll alone has only minor effects on Rac-GTP levels within the cell in comparison to ELMO: Dock180 (Figure 4a); Crkll may promote phagocytosis by other mechanisms, perhaps acting as a signaling submodule within the Crkll/CED-2, Dock180/CED-5, ELMO/CED-12, Rac/CED-10 pathway. However, Crkll was not able to enhance Rac-GTP levels when co-expressed with ELMO: Dock180 (Supplementary Figure 3), making it less likely that Crkll plays a direct role in regulation of Rac1-GTP in cells. This also suggested a likely Rac-independent role for Crkll. Crkll has been shown to stimulate cdc42 activation via the GEF N-WASP; ${ }^{32}$ overexpression of activating mutants of cdc42 have only mild effects on uptake of apoptotic targets, ${ }^{33,34}$ and we were unable to detect increased cdc42GTP levels following Crkll expression using the cdc42/Rac interactive binding (CRIB) domain assay (data not shown). Thus, it is unlikely that Crkll utilizes N-WASP and cdc42 to promote phagocytosis. Further, although CrkL has been shown to directly interact with WIP, ${ }^{35}$ we could not detect an interaction between Crkll and WIP.

An alternate possibility is that Crkll somehow recruits accessory proteins to the ELMO: Dock180 complex, which would in turn enhance the ability of ELMO:Dock180 to activate Rac. However, in cell-free exchange assays, Crkll immunoprecipitated from cells did not promote more exchange than ELMO:Dock180 alone, arguing against this interpretation. However, we found that although CrkII ${ }^{\mathrm{W} 170 \mathrm{~K}}$ or CrkII ${ }^{\text {W276K }}$ were unable to promote phagocytosis when expressed individually, they still cooperated with ELMO: Dock180 to promote uptake; mutations that removed both SH3 domains (CrkII ${ }^{\text {170KIW276K }}$ ) were no longer able to cooperate with ELMO: Dock180. This suggests that the two SH3 motifs act 'redundantly', not in binding to Dock180 but rather in ELMO : Dock180 complex 'activation'. However, the 
exact mechanism by which this occurs remains elusive; our experiments suggest that it may involve the small GTPase RhoG, as overexpression of a dominant-negative form, RhoG ${ }^{N 17}$ partially inhibits promotion of apoptotic target uptake by Crkll.

In summary, CED-2, CED-5, CED-12 and CED-10 were initially identified as part of the same genetic pathway in the nematode and subsequently determined to function in corpse removal in the mammalian context. We identify two evolutionarily conserved signaling submodules within this pathway, involving CED-2/CrkII, and CED-5/Dock180, respectively, that cooperate in promoting engulfment. This was identified in both enhancement of phagocytosis or cell migration using cell lines and in the context of a whole organism. We observe that RhoG might provide one point of crosstalk between the two modules. As the receptor upstream of the Crkll/Dock180/ ELMO/Rac module is unclear in both the worms and mammals, it is uncertain whether RhoG and Crkll would be 'activated' by the same receptor, or whether each signaling arm would be activated by individual upstream receptors that somehow forms a higher-order signaling network. Nevertheless, the identification of multiple inputs to the ELMO: Dock180 complex suggests a robust mechanism for the control of corpse removal.

\section{Materials and Methods}

Cell culture and reagents. $\mathrm{NIH} / 3 \mathrm{~T} 3, \mathrm{LR} 73$ and HeLa cells were cultured as described previously. ${ }^{24,36}$ Reagents used in this study: Phalloidin (Alexa 647) and highly cross-absorbed, conjugated secondary antibodies (Pacific Blue, Alexa 488, Alexa 555 and Alexa 647) (Molecular Probes, Invitrogen). The sources of the antibodies used were as follows: Anti-Crk (Transduction Laboratories, Lexington, KY, USA), Anti-Dock180 H-7 and anti-HA F-7 (Santa Cruz Biotechnology, SantaCruz, CA, USA), anti-HA 12CA5, anti-FLAG M2 and M5 (Sigma Chemical Co., St Louis, MO, USA), anti-ELMO1, ${ }^{8}$ anti-Crkll (Transduction Laboratories, KY, USA), anti-Rac1 (Upstate), anti-dynamin2 (C-18, Santa Cruz Biotechnology) and Anti-GFP (Santa Cruz Biotechnology). The plasmids encoding untagged and GFP-tagged $\mathrm{CrkII}^{\mathrm{WT}} \mathrm{CrkII}^{\mathrm{W} 170 \mathrm{~K}}, \mathrm{CrkII}^{\mathrm{W} 276 \mathrm{~K}}$ and CrkII ${ }^{\mathrm{W} 170 \mathrm{~K} / 276 \mathrm{~K}}$ (chicken) were kindly provided by Dr Ray Birge (UMDNJ-NJMS). The Dock180, DOHRS, Dock-ISP, Dock2, ELMO1 and RhoG plasmids have been described previously. $8,10,12$

Phagocytosis assay. LR73 cells were transiently transfected in triplicate with the indicated plasmids (either with GFP or GFP fusion proteins) in a 24-well plate. Twenty hours after transfection, the cells were incubated with $2 \mu \mathrm{m}$ carboxylatemodified red fluorescent beads, which mimic the negative charge on apoptotic cells and can serve as a simplified target, in serum-free medium (Sigma Chemical Co., St Louis, MO, USA). After $2 \mathrm{~h}$, the wells were then washed twice with cold PBS, trypsinized, resuspended in cold medium (with $1 \%$ sodium azide) and analyzed by two-color flow cytometry. The transfected cells were recognized by their GFP fluorescence. Forward and side-scatter parameters were used to distinguish unbound beads from cells. For each point, 45000 events were collected and the data was analyzed using Cell Quest and/or FlowJo software. As shown previously, ${ }^{8}$ the majority of double-positive cells scored in the FACS assay represents particles engulfed by transfected cells or particles in the process of engulfment, and do not represent beads simply bound to the cell surface. $\mathrm{NIH} / 3 \mathrm{~T} 3$ cells were incubated with $2 \mu \mathrm{m}$ beads in serum-free medium for $1 \mathrm{~h}$ 48-h after nucleofection (Amaxa).

In vitro GEF assay. The radioactivity-based in vitro GEF assay was performed as described previously. ${ }^{8} 293 \mathrm{~T}$ cells in $10 \mathrm{~cm}$ dishes were transfected with the indicated plasmids and the cell lysates were immunoprecipitated with anti-FLAG or anti-GFP antibodies conjugated beads. The precipitated proteins were quantitated via Western blotting and analyzed for Rac GEF activity. The presence of GEF activity was revealed by loss of radioactivity bound to $\operatorname{Rac}\left(\alpha{ }^{-32} \mathrm{P}-\mathrm{GTP}\right)$ (due to the exchange reaction). Relative GEF activity was presented as the percentage of remaining radioactivity bound to Rac, with samples from mock-transfected 293T cell set at $100 \%$.
Immunoprecipitations and immunoblotting. 293T cells were transiently transfected with $2 \mu \mathrm{g}$ of Crkll constructs, $2 \mu \mathrm{g}$ of ELMO-GFP or $10 \mu \mathrm{g}$ of Dock180, Dock2 and Dock180-DOHRS plasmids (which were less well expressed). LR73 cells were plated in six-well plates and transfected using Lipofectamine 2000 as described above. After $36 \mathrm{~h}$, the cells were lysed (in 1\% Triton X-100, $50 \mathrm{mM}$ Tris and $150 \mathrm{mM} \mathrm{NaCl}$ ) and immunoprecipitated using antiFLAG antibody directly coupled to sepharose (clone M2) (Sigma Chemical Co.).

RNA interference. siRNAs to mouse Crk were purchased from Dharmacon (cat no.1 L-061117-00-0005) as a combination of four siRNAs. Concentration of siRNA used in experiments is total concentration of siRNA rather than concentration of each individual siRNA. Control siRNA no. 1 was also purchased from Dharmacon (cat no. D-001210-01-20). siRNAs experiments were carried out in $\mathrm{NIH} / 3 \mathrm{~T} 3$ fibroblasts, rather than LR73 cells, due to the poor knock-down of endogenous Crkll achieved in LR73 cells. siRNAs $(0.4 \mu \mathrm{g})$ were introduced into $\mathrm{NIH} / 3 \mathrm{~T} 3$ fibroblasts using the Amaxa Nucleofector I (program U-30) and Nucleofector kit R (Amaxa, Germany) according to manufacturer's directions. Transfection efficiency was $>95 \%$ as measured by expression of a GFP reporter in these cells (data not shown).

Immunofluorescence staining. HeLa cells were plated on Labtek II slides and transfected with Lipofectamine 2000 as described previously. ${ }^{36}$ At harvest, slides were washed $2 \times$ with $1 \times$ TBS then fixed in $3 \%$ formaldehyde for $7 \mathrm{~min}$, then permeabilized for $2^{\prime}$ with TBS $+0.2 \%$ Triton X-100 (Fisher). Cells were then blocked with clarified milk and subsequently stained with appropriate antibodies. Immunostaining was performed sequentially with IgG-specific secondary antibodies.

C. elegans migration and phagocytosis assays. DTC migration and engulfment were scored as previously described. ${ }^{12}$ The indicated Dock180 and CED-5-coding sequences were subcloned into the transgenic vector driven by the Peft-3 promoter. Clones were co-injected with either Plim-7::gfp (for scoring DTC migration) or pTG96 (Psur-5::gfp, for scoring engulfment defect). Injected hermaphrodites were picked and allowed to have progeny. Transgenic progeny were moved to individual plates and allowed to grow. Worms that transmitted the array were kept and assayed for expression (brightness of GFP signal) and transmittance of the array. Strains with the highest transmittance/GFP signal were kept for further analysis. Worms were maintained at $20^{\circ} \mathrm{C}$ as described previously. ${ }^{10}$ Clean transgenic worms were moved to a large plate that was seeded with OP50 bacteria and allowed to propagate one generation. For DTC migration, worms were then scored under a Zeiss M2Bio-dissecting microscope equipped with epifluorescence; worms with a gonad that deviated from the standard U-shaped tube was scored as migration defective. Engulfment defect was scored in the L1 head as described previously. ${ }^{37}$

Acknowledgements. We thank Dr. Ray Birge for kindly providing us GFPCrkll plasmids, Jan Redick and Christie Davis of the Advanced Microscopy Core Facility at University of Virginia for help and advice on confocal microscopy, and Costi Sifri, Gari Stergiou, Cheng-Wen Su, Cynthia Grimsley and members of the Ravichandran lab for helpful discussions. This work was supported by NIH grants to KSR and grants from the EU APOCLEAR, the Ernst Hadorn Foundation, and the Swiss National Science Fund to MOH. JMK is a recipient of an Arthritis Foundation Postdoctoral Fellowship.

1. Henson PM, Hume DA. Apoptotic cell removal in development and tissue homeostasis. Trends Immunol 2006; 27: 244-250.

2. Kinchen JM, Hengartner MO. Tales of cannibalism, suicide, and murder: Programmed cell death in C. elegans. Curr Top Dev Biol 2005; 65: 1-45.

3. Mevorach D. The immune response to apoptotic cells. Ann N Y Acad Sci 1999; 887: 191-198.

4. Scott RS, McMahon EJ, Pop SM, Reap EA, Caricchio R, Cohen PL et al. Phagocytosis and clearance of apoptotic cells is mediated by MER. Nature 2001; 411: 207-211.

5. Tan EM. Meaning of autoantibodies in lupus-like syndromes. Lupus 1994; 3: 483-485.

6. Grimsley C, Ravichandran KS. Cues for apoptotic cell engulfment: eat-me, don't eat-me and come-get-me signals. Trends Cell Biol 2003; 13 (12): 648-656.

7. Gardai SJ, Bratton DL, Ogden CA, Henson PM. Recognition ligands on apoptotic cells: a perspective. J Leukoc Biol 2006; 79: 896-903

8. Brugnera E, Haney L, Grimsley C, Lu M, Walk SF, Tosello-Trampont AC et al. Unconventional Rac-GEF activity is mediated through the Dock180-ELMO complex. Nat Cell Biol 2002; 4: 574-582. 
9. Cote JF, Vuori K. Identification of an evolutionarily conserved superfamily of DOCK180related proteins with guanine nucleotide exchange activity. J Cell Sci 2002; 115 (Part 24): 4901-4913.

10. Gumienny TL, Brugnera E, Tosello-Trampont AC, Kinchen JM, Haney LB, Nishiwaki K et al. CED-12/ELMO, a novel member of the Crkll/Dock180/Rac pathway, is required for phagocytosis and cell migration. Cell 2001; 107: 27-41.

11. Grimsley CM, Kinchen JM, Tosello-Trampont AC, Brugnera E, Haney LB, Lu M et al. Dock180 and ELMO1 proteins cooperate to promote evolutionarily conserved Racdependent cell migration. J Biol Chem 2004; 279: 6087-6097.

12. deBakker CD, Haney LB, Kinchen JM, Grimsley C, Lu M, Klingele D et al. Phagocytosis of apoptotic cells is regulated by a UNC-73/TRIO-MIG-2/RhoG signaling module and armadillo repeats of CED-12/ELMO. Curr Biol 2004; 14: 2208-2216.

13. Katoh $\mathrm{H}$, Negishi M. RhoG activates Rac1 by direct interaction with the Dock180-binding protein Elmo. Nature 2003; 424: 461-464.

14. Hasegawa H, Kiyokawa E, Tanaka S, Nagashima K, Gotoh N, Shibuya M et al. DOCK180, a major CRK-binding protein, alters cell morphology upon translocation to the cell membrane. Mol Cell Biol 1996; 16: 1770-1776.

15. Reddien PW, Horvitz HR. CED-2/Crkll and CED-10/Rac control phagocytosis and cell migration in Caenorhabditis elegans. Nat Cell Biol 2000; 2: 131-136.

16. Wu YC, Horvitz HR. C. elegans phagocytosis and cell-migration protein CED- 5 is similar to human DOCK180. Nature 1998; 392: 501-504.

17. Wu YC, Tsai MC, Cheng LC, Chou CJ, Weng NY. C. elegans CED-12 acts in the conserved crkll/DOCK180/Rac pathway to control cell migration and cell corpse engulfment. Dev Cell 2001; 1: 491-502.

18. Zhou Z, Caron E, Hartwieg E, Hall A, Horvitz HR. The C. elegans PH domain protein CED-12 regulates cytoskeletal reorganization via a Rho/Rac GTPase signaling pathway. Dev Cell 2001; 1: 477-489.

19. Wu YC, Cheng TW, Lee MC, Weng NY. Distinct rac activation pathways control Caenorhabditis elegans cell migration and axon outgrowth. Dev Biol 2002; 250: 145-155.

20. Lundquist EA, Reddien PW, Hartwieg E, Horvitz HR, Bargmann Cl. Three C. elegans Rac proteins and several alternative Rac regulators control axon guidance, cell migration and apoptotic cell phagocytosis. Development 2001; 128: 4475-4488.

21. Cote JF, Motoyama AB, Bush JA, Vuori K. A novel and evolutionarily conserved Ptdlns $(3,4,5) \mathrm{P}_{3}$-binding domain is necessary for DOCK180 signalling. Nat Cell Biol 2005 7: 797-807.

22. Sanui T, Inayoshi A, Noda M, Iwata E, Stein JV, Sasazuki T et al. DOCK2 regulates Rac activation and cytoskeletal reorganization through interaction with ELMO1. Blood 2003 102: $2948-2950$
23. Nishihara $\mathrm{H}$, Kobayashi $\mathrm{S}$, Hashimoto $\mathrm{Y}$, Ohba F, Mochizuki N, Kurata $T$ et al. Nonadherent cell-specific expression of DOCK2, a member of the human CDM-family proteins. Biochim Biophys Acta 1999; 1452: 179-187.

24. Tosello-Trampont AC, Brugnera E, Ravichandran KS. Evidence for a conserved role for CRKII and Rac in engulfment of apoptotic cells. J Biol Chem 2001; 276: 13797-13802.

25. Feller SM. Crk family adaptors-signalling complex formation and biological roles. Oncogene 2001; 20: 6348-6371.

26. Kiyokawa $E$, Hashimoto $Y$, Kurata $T$, Sugimura $H$, Matsuda M. Evidence that DOCK180 up-regulates signals from the Crkll-p130(Cas) complex. J Biol Chem 1998; 273: 24479-24484.

27. Akakura S, Kar B, Singh S, Cho L, Tibrewal N, Sanokawa-Akakura R et al. C-terminal SH3 domain of Crkll regulates the assembly and function of the DOCK180/ELMO Rac-GEF. J Cell Physiol 2005; 204: 344-351.

28. Kuriyan J, Cowburn D. Modular peptide recognition domains in eukaryotic signaling Annu Rev Biophys Biomol Struct 1997; 26: 259-288.

29. Lu M, Kinchen JM, Rossman KL, Grimsley C, deBakker C, Brugnera E et al. PH domain of ELMO functions in trans to regulate Rac activation via Dock180. Nat Struct Mol Biol 2004 11: 756-762

30. Kobayashi S, Shirai T, Kiyokawa E, Mochizuki N, Matsuda M, Fukui Y. Membrane recruitment of DOCK180 by binding to Ptdlns $(3,4,5) P_{3}$. Biochem J 2001; 354 (Part 1): 73-78.

31. Zhou Z, Hartwieg E, Horvitz HR. CED-1 is a transmembrane receptor that mediates cell corpse engulfment in C. elegans. Cell 2001; 104: 43-56.

32. Tang DD, Zhang W, Gunst SJ. The adapter protein Crkll regulates neuronal WiskottAldrich syndrome protein, actin polymerization, and tension development during contractile stimulation of smooth muscle. J Biol Chem 2005; 280: 23380-23389.

33. Tosello-Trampont AC, Nakada-Tsukui K, Ravichandran KS. Engulfment of apoptotic cells is negatively regulated by Rho-mediated signaling. J Biol Chem 2003; 278: 49911-49919.

34. Nakaya M, Tanaka M, Okabe Y, Hanayama R, Nagata S. Opposite effects of rho family GTPases on engulfment of apoptotic cells by macrophages. J Biol Chem 2006; 281 8836-8842.

35. Sasahara Y, Rachid R, Byrne MJ, de la Fuente MA, Abraham RT, Ramesh N et al. Mechanism of recruitment of WASP to the immunological synapse and of its activation following TCR ligation. Mol Cell 2002; 10: 1269-1281.

36. Grimsley CM, Lu M, Haney LB, Kinchen JM, Ravichandran KS. Characterization of a nove interaction between ELMO1 and ERM proteins. J Biol Chem 2006; 281: 5928-5937.

37. Kinchen JM, Cabello J, Klingele D, Wong K, Feichtinger R, Schnabel H et al. Two pathways converge at CED-10 to mediate actin rearrangement and corpse removal in $C$. elegans. Nature 2005; 434: 93-99. 